\title{
Design and Implement of Wireless Virtual Classroom
}

\author{
Auday Jamal Fawzi \\ Faculty of Computer and I.T, Nawroz University, Dohuk, Iraq
}

\begin{abstract}
In this research a synchronous virtual classroom are designed. The proposed application is in the field of distance education and wireless technology. A wireless platform is developed to design two programs using Ad-Hoc technique that performs the task of virtual classroom for teachers and students to establish a classroom dynamically irrespective of location. By taking lessons in a multimedia learning environment, it is expected that students will improve their learning performance with perhaps less attendance in a physical classroom and they gain the flexibility of being able to learn at their own convenience
\end{abstract}

Keywords: Client/Server Computing, Distance Learning, Virtual Classroom, Wireless Network.

\section{Introduction}

The rapid expansion of information and communication technologies opens new paths towards the implementation and delivery of distance learning. More specifically, the development of collaboration tools acts as a basis for the design and implementation of "Wireless Virtual Classrooms" to support flexible educational and training systems [1].The goal of this project is to develop a wireless platform for teachers and students, to build an Ad-Hoc classroom, and to develop an information technology to provide teachers with teaching aids, and to create an environment for students to self motivated learning. Drashti Shrimal and et.al. (2013) [2], designed concept of e-learning and virtual classroom which include (students forum, live chat, whiteboard, file upload/download, and one way video conferencing. While N. K. Subramaniam and et.al. (2011) [3], explores the use of the virtual classroom which has been created in $m y V L E$, a learning management system. The virtual classroom in $m y V L E$ is an asynchronous-based online learning environment that delivers course materials to learners and provides collaboration and interaction using an asynchronous-based forum as the main platform to support the learners' independent study. It also provides a learning environment with learning tools, learning materials, opportunities for contextual and collaborative discussions, and individual learning and assessment. Erika Pigliapoco and et.al. (2006) [4], present an enhanced chat room developed to recreate the environment of a physical university classroom within a learning management system. The enhanced chat tool, called LoL Classroom, its presentation is taken as an opportunity for proposing a more general discussion about the usage of synchronous textual interactions in computer-mediated learning environments inspired to a constructivist teaching model.

\section{Virtual Classroom Overview}

A Virtual Classroom is a teaching and learning environment constructed in software, which supports collaborative learning among students who participate at times and places of their choosing, through computer networks.

A virtual classroom is a real-time electronic classroom where instructors can meet their students and share ideas on a particular subject. The program offers a class-specific home page for the on-line class which could include teaching materials, syllabus, examination, a white board, assignment, student information and access to the chat room. The on-line class can be synchronous, asynchronous, or a combination of both $[5,6]$.

\section{Learning Models}

Learning model is divided into four categories [7]:

\section{A. Learn from information}

This is basic knowledge transfer, ideal for new product launches, corporate strategy, or organizational announcements. These are materials where learners can quickly and simply get the information they need, for example informational web pages, online help, streaming audio, videos, web books and web lectures.

\section{B. Learn from interaction}

Interaction is between the learner and a computer system not with other learners or instructors, these are selfpaced activities, the materials are specially designed for learning purposes, for example interactive games, simulation programs, quizzes, tests, and assignments corrected by the system. 


\section{Learn from collaboration}

Communication is facilitated by technology, which means that learners and instructors can work from different places. Collaboration tools enable learner-to-learner communication, group learning, or learnerinstructor communication, combining all these can form a sense of learning community, Collaborative eLearning can be asynchronous or synchronous.

- Asynchronous collaboration: Asynchronous collaboration tools do not require learners and instructors to be online at the same time to communicate with each other. Messages, questions, or assignments can be posted and answered at any moment. Others can read them and respond when they are online.

- Synchronous collaboration: Synchronous collaboration provides online communication in real-time. Instructors and learners come together electronically at a given time, without leaving the workplace, they use tools such as chat, audio, video, and whiteboards.

\section{Learn from co-location}

This category represents high-level learning activities that do not rely on technology, there will always remain situations where traditional classroom-based training will be most effective; where learners need to get together with an expert, and physically interact with others or with objects, for example traditional classroom.

\section{Virtual Classroom Requirements}

The narrow view of the virtual classroom is that it is a room with computers and multimedia displays. The broader view is that it is a virtual environment that supports many processes of classroom education. But to provide such an environment the classroom must have some combination of the following elements[8]:

- A server computer for the instructor.

- A multimedia system capable of presenting a variety of types of information (e.g., text, graphics, animation, audio, and video).

- A database of educational materials within the classroom.

- A client computer for each student.

- A local area network that allows communication among all of the workstations.

- A system that provides storage, sharing, and transfer of documents.

- A telecommunications system to link the classroom to external educational resources.

The fully integrated electronic classroom will have all of these elements and perhaps more. There will be many transitional and semi-integrated classrooms that will only be able to support some elements. For example, there will be:

(a) Classrooms that allow the instructor to project multimedia displays and simulations during lectures but not allow the students to have hands on experience with the materials;

(b) Classrooms that provide workstations for all of the students but do not provide an ability to share results and display them to the class as a whole; and

(c) Classrooms that are integrated within themselves but are not networked to the outside world.

In each case instructors need to understand the enhanced instructional capabilities available through the technology and use them effectively in combination with traditional approaches.

\section{Wireless Network Technology}

Wireless technology has changed the way to connect to internet, local and remote computers, it has enabled the implementation of imagination beyond the limits. There are two common modes to connect the WiFi for connectivity for accessing internet or other computer for file and printer sharing. The most common modes for Wi-Fi connectivity are infrastructure mode and Ad hoc Mode [9].

\section{Infrastructure Mode}

Infrastructure is one of the two methods for connecting to wireless networks with Wi-Fi enabled devices. These devices are connected to wireless network with the help of Access point (AP). Wireless Access Points are usually routers or switches which are connected to internet by Ethernet port. Wireless Access points are always required for infrastructure mode of wireless networking. It is necessary to use SSID while configuring AP, where SSID should be known to client computers to connect WLAN. SSID is basically security key which help prevent unauthorized access to WLAN. The Access point is then connected to wire network (Internet) to provide wireless internet connectivity to clients. Multiple access points can be added in the WLAN, this increases the reach of infrastructure for supporting many number of wireless clients [9]. 


\section{Ad-Hoc Mode}

This mode is known as ad hoc mode. By using this mode, devices are capable for communicating directly with each other. No Access point (routers / switches) is required for communication between devices and all devices in the range connect in peer to peer communication mode.

The disadvantage associated with infrastructure mode is extra cost to for Access points, routers and switches. Compared to the ad-hoc wireless networks, infrastructure mode has much more stability, scalability, centralized security management and improved reach. Ad hoc mode is best used for small number of devices which are physically present in close proximity with each other and as the number of devices grows performance of network suffers. Disconnections of random device may occur time to time. There is another limitation associated with ad hoc mode that is, ad hoc mode networks cannot bridge to wire LANs and cannot access internet without installing special gateways [10].

\section{The Proposed Virtual Classroom System}

A real-time interactive virtual classroom allows a remote participant to receive a live class feed, as well as to interact in a live class by asking questions with real-time text and audio, and sharing activities.

\section{Proposed Hardware System}

The goal is to design a network system that makes a flexible, quick and easy communication for the students to use the virtual classroom. The next paragraph explains in details how this system works.

In this system the server, with two access point are connected to the router by a cable, and wireless clients that interconnected to the network through the access point that functions as a wireless bridge between the server and the clients to coverage a wide wireless network area (more than two access point can connected to increase the coverage area) as shown in figure (1). The wireless clients are easily connected to the network where gets its IP address automatically from the router at boot up.

The server has share resources and multimedia files with the clients, in addition to the network resources such as internet access and a printer.

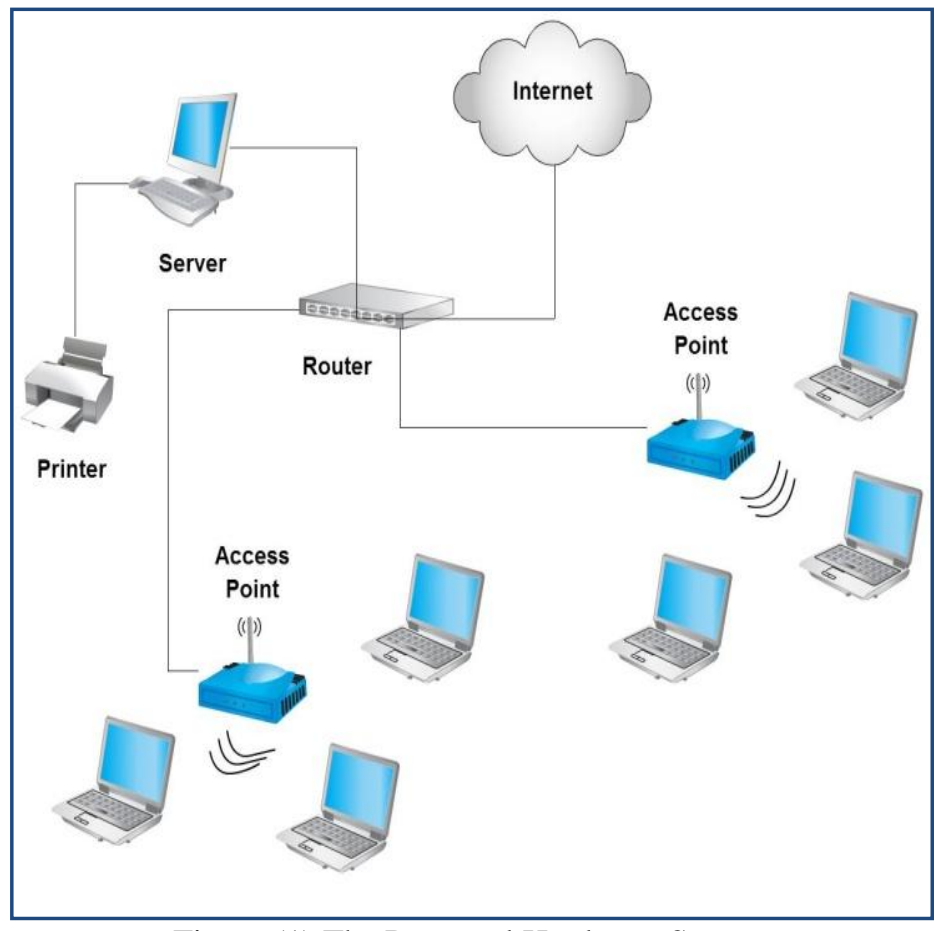

Figure (1) The Proposed Hardware System

\section{Proposed Software System}

Two programs are designed to perform the virtual classroom system. The first, works as instructor program (inside server) while the second, works as student program (inside client).

The virtual classroom software has the ability of interactive multimedia communications between student and instructor as shown in figure (2) using various tools (Text Message, Audio Conference, White Board, and View Video). Instructor has the ability to supervise the student's work and able to monitor student's progress during the course. 


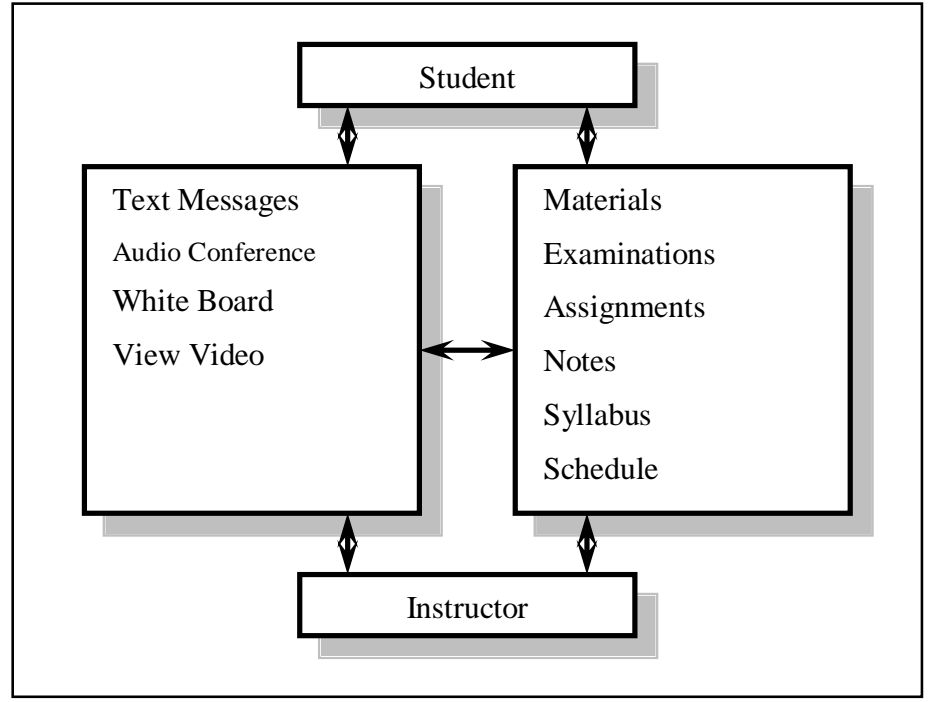

\section{A. Program Functions}

Figure (2) Proposed Program Functions

The program consists of several functions that spread on the home screen environment as shown in figure (3), most of these functions are connected to a database to derive its information like student information, student attendance, teaching materials, syllabus, lectures schedule, and examination questions and answers tables. Each function was designed to perform specific operation, these functions, including the following:

- Material: The teaching materials consist of all of the written materials, collected notes and plans that exist at the beginning of the course.

- Whiteboard: The Whiteboard function enables the teacher to represent different types of information, it is a feature of the Virtual Classroom that simulates an actual whiteboard in a classroom. The benefit of using whiteboard that it requires less bandwidth compared to screen sharing.

- Web: This function enables the students to search the web.

- Notes: The Notes function provides access to the individual notes written by the students.

- Tool: The tool window provides many applications that assist the student to ease his work, it allows the student to quickly access many necessary applications.

- Voice Conference: The conference function enables the student to make a live conversation with the teacher. All students can hear the teacher, but only those students that the teacher allows to talk can make a conversation.

- Text Chat: Live text can be exchanged between teacher to many students or students between each other.

- Video: Provides a way to open the video record captured by the teacher by his webcam.

- Exam: This function enables the teacher to make online examination with the controls to start and end the exam. At the end of the time the server force the clients to stop and store the student's answer in the server.

- Assignment: Assignment function is electronically distributed and collects the answer of homework.

- Schedule: The schedule function provides daily schedule data.

- Syllabus: The syllabus function promotes the idea of the class organized around daily topics.

- Alert: Students will be alerted for instant chat, assignment, exam, incoming voice chat and change in whiteboard content.

- Student Profile: The Student profile function provides student's information like his name, password, class, email, marks, photo picture and other personal information.

- Monitoring: Monitoring function used to show what students are doing at their computers.

- Site Map: The Site map function provides a plan represent each computer connected to the software or not with its IP address and the name of the students' login.

\section{B. Establish a Network Connection}

The client connected to the server through a network socket which provides easy access to TCP and UDP network services. The first consideration is whether to use the TCP or the UDP protocol. The difference between the two lies in their connection state, where the TCP protocol is a connection-based protocol, and the user must establish a connection before proceeding, while the UDP protocol is a connectionless protocol, and the transaction between two computers is like passing a note. For this reason, the TCP protocol is used in this research. 
The Windows Sockets API (WSA), which shortened to Winsock, is a technical specification that defines how Windows network software should access network services, where the Winsock allows creating and maintaining a connection to a remote computer, using this connection, both computers can stream data between themselves. In a client side, a server computer's name or IP address must be known, as well as the port on which it will be "listening", while in a server side, a port on which to listen is set. When the client requests a connection, the connection request will occur, and to complete the connection, server accepts the connection request. Once a connection has been made, either computer can send and receive data. The server can connected to more than on client using a multi socket technique.

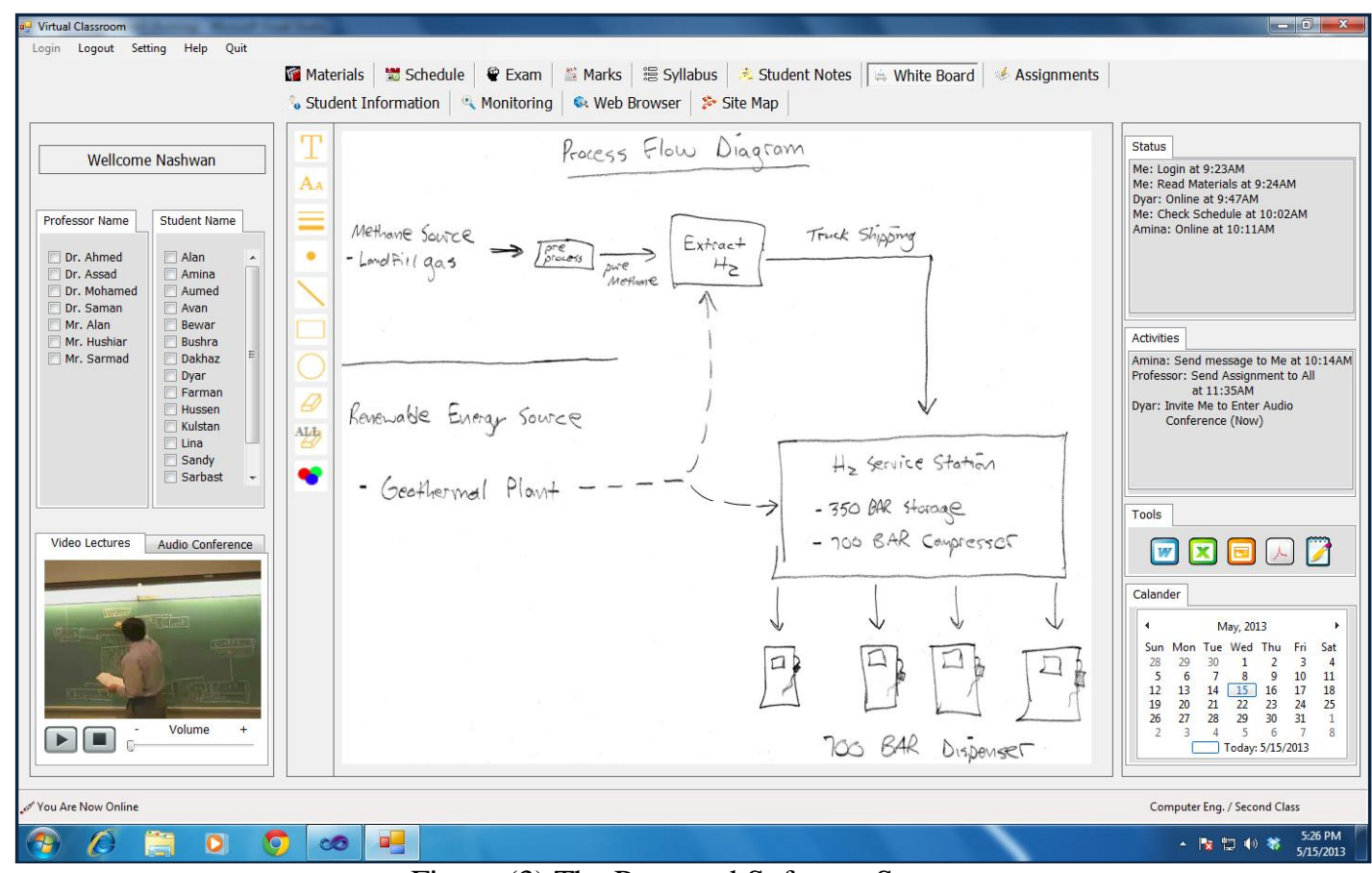

Figure (3) The Proposed Software System

\section{Examples on Program Functions}

We will take two functions and describe it in brief, the first one is the connection between the client and the server, when class start, the instructor select his name from the instructors list shown in left side of the program after that the program make a new socket connection with an appropriate IP address and port number, waiting for the client to request a connection. In the client side the student select his name from the students list, enter his password and select the instructor name from the instructors list that he want to connect to, after password verification the program make a socket connection depending on IP address and port number of the server and send a connection request to the server which already in listening mode, the server send back a connection accept that leads to connect the server with the clients, as shown in figure (4).

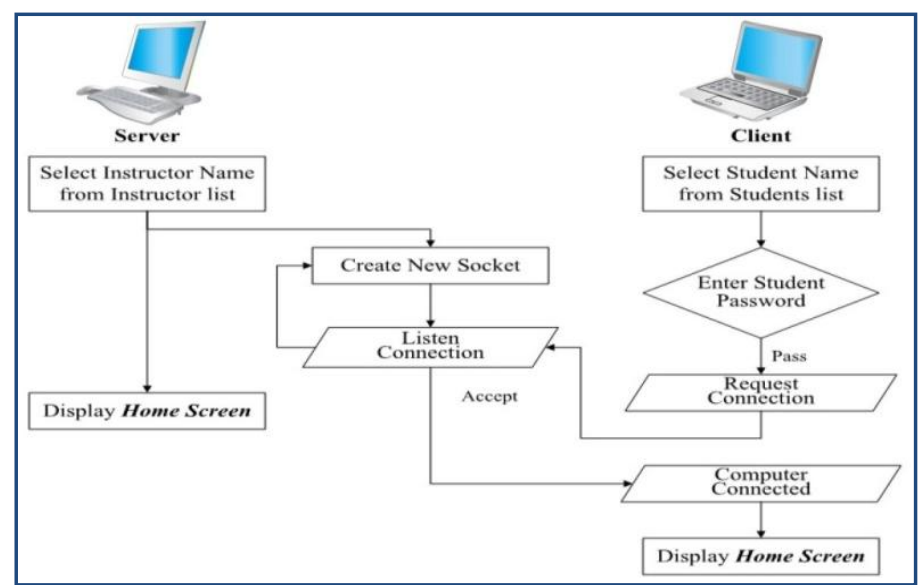

Figure (4) Procedure of client/server connection 
The second is the examination function, when the instructor decide to make an examination, he select the examination tab from the home screen then select the exam and the type of questions (multi-choice or explanation questions) then select start the exam button, the server inform the clients that the examination was start, in this time the clients pause all it work, open the examination tab automatically and make a call to get the questions from the server examination database. At this time the student will answer the questions, there are two possibilities, the first is the student finish his answer before the end time then he can save his answer and quit the tab, while the second if the exam time is finished before the student finish his answer, the program prevent the student from further writing in the answer space, save the answer, and in both cases the client send the answer to the server to save it under the private student portion. Figure (5) represent the operation of examination function between client/server programs.

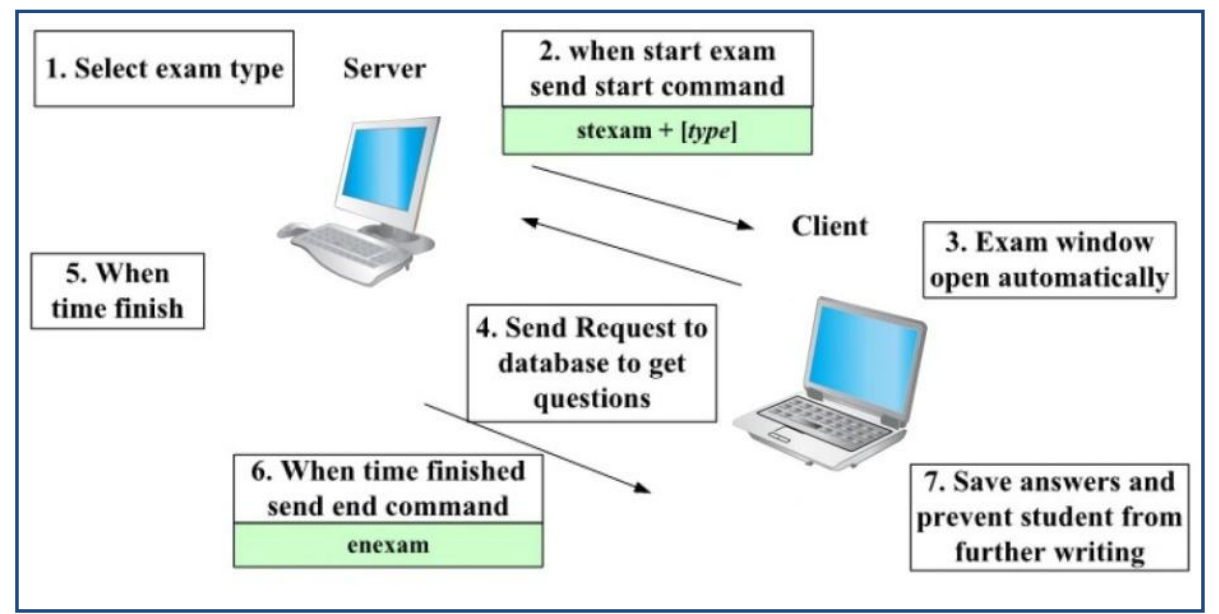

Figure (5) The operation of exam function

\section{Conclusion}

This paper presents a design and implement of a wireless virtual classroom system, must functions presented in previous classroom system are implemented. It is a process of communication between instructor and students. The instructor leads the class and monitor student's progress during the course, he can add new students, add various teaching materials, write on whiteboard, participate with students through text chat or voice conference, make an examination, put student evaluation, check student assignments and monitoring the whole system. While the student can read the teaching materials, chat in text or voice with the instructor or other students, answer examination questions, view teaching video provided by the instructor, and use some available resources like web, powerpoint, excel sheets and paint, this allow the students to immerse themselves in friendly environments that make the learning process more enjoyable.

Since this network connecting through a wired and wireless LAN, and does not depend on internet in its connection, which increase the efficiency of the system, where the internet only used to get external teaching resources.

\section{References}

[1] Apostolos, P., Demosthenes, S., Dimitris, P., \& Anastasios, M.(2003). The Design and Implementation of a Networked Virtual Classroom: A case study in the area of Fluids Physics.Journal of Information Technology Impact, Vol. 3, No. 3, pp. 143-157.

[2] Drashti S., Shraddha S., \& Deepika J. (2013). Virtual Class Management. International Journal of Computer and Electronics Research, Vol. 2, No. 2, pp. 143-147.

[3] Nantha K. S., \& Maheswari K. (2011). The Virtual Classroom: A Catalyst for Institutional Transformation. Australasian Journal of Educational Technology, Special issue 8, pp. 1388-1412.

[4] Erika P., Giovanni T., Massimo M., \& Alessandro B. (2006). LoL Classroom: A Virtual University Classroom Based on Enhanced Chats. ICL2006 Conference in Austria, 27-29 September.

[5] Starr, R. H. (1995). The Virtual Classroom: Learning Without Limits via Computer Networks (2nd ed.). NJ: Ablex Publishing Corporation.

[6] Wendy, G. C. \& Cindy,H. (2009). Designing for the Virtual Classroom (Issue: 0911). USA: American Society for Training and Development Press.

[7] Mike E., David B., Balaka G., Dan N., \& Phil S. (2003). Using IBM Lotus Virtual Classroom: A Best Practices Guide to e-Learning (2nd ed.). USA: IBM Corporation.

[8] Kent, L. N. (1997). An Introduction to Electronic Education and Hyper Courseware. Retrieved June 25, 1997, from http://www.lap.umd.edu/soc/sochome.html.

[9] Steve R. (2011). Wireless Networking Technology: From Principles to Successful Implementation (2nd ed.). UK: Elsevier.

[10] Subir K. S., T. G. Basavaraju \& C. Puttamadappa (2013), Ad Hoc Mobile Wireless Networks: Principles, Protocols, and Applications (2nd ed.). USA: CRC Press. 


\section{Biography}

Auday Jamal Fawzi (M.Sc.'01-Ph.D.'07). Born in Baghdad, Iraq, 1977. Got his B.Sc. in Computer and Control Engineering from Baghdad, University of Technology 1999, his M.Sc. in Electrical Engineering from Electromechanical Department from the same university 2001, and Ph.D. from the same department 2007. Currently working as Lecturer and Dean of the Computer and Information Technology Faculty in Nawroz University, Duhok City, Kurdistan Region of Iraq. Major Fields of studies are logic design, build software applications, and security.

He worked as Assistant Lecturer in the Computer and Control Engineering Department, Electromechanical Engineering Department of in University of Technology, Baghdad, and Lecturer in Computer Science Department and Computer and Communication Engineering Department in Nawroz University. Published many papers in the Scientific Journal of Nawroz University and a paper in ASME Journal. Dr. Eng. Fawzi is a member in Engineering Syndicate of Kurdistan Region of Iraq, and a member in the Iraqi Engineering Syndicate. 